\title{
STUDY ON 3D SOLID RECONSTRUCTION FROM 2D VIEWS BASED ON INTELLIGENT UNDERSTANDING OF MECHANICAL ENGINEERING DRAWINGS
}

\author{
Jianping Liu ${ }^{1,2}$, Bangyan $\mathrm{Ye}^{1}$, Xiaohong $\mathrm{Wu}^{3}$, Miaoan Ouyang ${ }^{2}$ \\ 'College of Mechanical Engineering, South China Univesity of Technology, Guangzhou, \\ 510640, China; Email: lip68@tom.com. ${ }^{2}$ Department of Mechatronics, Foshan Polytechnic \\ College, Foshan, 528237, China; ${ }^{3}$ College of Information, South China Agricultural \\ Univesity, Guangzhou, 510640, China.
}

Abstract: This study presents a new algorithm of 3D reconstruction to obtain automatically 3D solid from mechanical engineering drawings. Main steps of the algorithm are as follows: first, reconstruct multiple views from dxf-type files; second, recognize primitives feature and combined relationship between primitives by engineering semantics understanding and loops searching on views; third, partition and reconstruct body primitives; last, all reconstructed primitives are assembled into a goal solid by Boolean operation. The algorithm adopts integrated reconstruction techniques to enlarge the figuration domain of reconstructed objects. And it uses case-based reasoning to set up a selflearning mechanism of feature recognition. It is implemented at the desktop of AutoCAD 2004 by using Object ARX 2002 and VC++6.0. The results show that utility scope and reconstruction speed are improved when using the new algorithm.

Key words: 3D reconstruction; mechanical engineering drawings; primitive; feature recognition; engineering semantics understanding.

\section{INTRODUCTION}

$3 \mathrm{D}$ reconstruction from mechanical engineering drawings is a method of automatic constructing $3 \mathrm{D}$ solid objects. It is the pivot part of the system converting 2D paper-based engineering drawings into 3D CAD model files. It can provide $3 \mathrm{D} \mathrm{CAD}$ model for application of advanced manufacturing

This project is supported by the Key Item of Science and Technology of Guangdong, China under the grant No.2002A1020103 and No.2005C1030038.

Please use the following format when citing this chapter:

Liu, Jianping, Ye, Bangyan, Wu, Xiaohong, Ouyang, Miaoan, 2006, in International ederation for Information Processing (IFIP), Volume 207, Knowledge Enterprise: Intelligent Strategies In Product Design, Manufacturing, and Management, eds. K. Wang, Kovacs G., Wozny M., Fang M., (Boston: Springer), pp. 704-709. 
technology such as CAM, CAPP, CAE, PDM, CIMS, VM, RP\&M, etc. Its application can not only save tremendous time and labor but also broaden the domain of computer vision from perspective images understanding to projective graphics understanding.

Idesawa first presented this issue in his paper "A system to generate a solid figure from three view" in $1973^{[1]}$. Various algorithms starting from three views have been published in the literature since then. Among them, boundary representations (B-rep), constructive solid geometry (CSG) and the approach-based algorithm are typical algorithms and most of other algorithms are improvements of the typical ones ${ }^{[2]}$. Main steps of the B-Rep algorithm ${ }^{[3]}$ are as follows: a wire-frame that is composed of 3D vertices and $3 \mathrm{D}$ edges is first recognized from the input orthographic views. Then, candidate faces are found from all these 3D edges. Pseudo elements are deleted by certain criteria and all true faces are assembled to form a final solid. The CSG oriented approach ${ }^{[4]}$ assumes that each $3 \mathrm{D}$ object can be built from certain primitives in a hierarchical manner. It selects a 2D loop as a base and generates the $3 \mathrm{D}$ primitives by matching their corresponding $2 \mathrm{D}$ patterns in each view, then assembles reconstructed primitives to form a goal solid. The approach-based algorithm ${ }^{[5]}$ is similar to cutting mechanical part. Its main steps are as fellows: First, a reference solid is generated by translational-sweeping and Boolean intersection operation. Then, the difference solid is reconstructed by comparing the views between the reference solid and the result solid. Finally, by subtracting the difference solid from the reference solid one by one and testing the residual solid by views, the goal solid can be finally obtained. Although 3D reconstruction from $2 \mathrm{D}$ orthographic views has been studied for over three decades, the present algorithms are still far from the desired goal of a general solution and practical application ${ }^{[6]}$. The main problems of geometric reconstruction are as follows: 1) Reconstructed objects are usually three orthographic views, where there are no centerlines, no dimension, no cutaway views, no sectional views and no assistant views and so on. 2) For B-Rep, its reconstruction efficiency is low and it is easy to generate pathological cases; For CSG, it is difficult to deal with complex figuration because limited pre-defined primitives patterns restrict its applicability; For the approach-based algorithm, there are a great deal of operations and complicated calculation in 3D space. 3) Using single reconstruction technique leads to limited figuration domain of reconstructed objects. 4) Present algorithms of primitive feature recognition are difficult to deal with intersection and complex primitive features.

Researchers think limits of traditional algorithms based on geometric reconstruction cause the less progress on $3 \mathrm{D}$ reconstruction research. In 
order to solve existing problems and seek a new breakthrough, they attempt to use the technique of engineering semantics understanding to recognize primitive feature. In 1984, Yashiura ${ }^{[7]}$ first uses the information conveyed by dimension annotation to recognize primitives. In 1992, Joseph ${ }^{[8]}$ makes feature recognition easier by analyzing the semantics of sectional views. In China, Lu ${ }^{\left[{ }^{[0]}\right.}$ first uses the semantics information conveyed by semi-section or full section views to reconstruct $3 \mathrm{D}$ solid. In order to technique practicability of 3D reconstruction, we take practical mechanical engineering drawings which contain section views, local views and have dimensions as reconstructed objects and present a new algorithm based on engineering semantics understanding.

\section{NEW ALGORITHM OF 3D RECONSTRUCTION}

The key problem of engineering drawings understanding is how to recovery the losing information of geometry and topology at the projective depth direction. Experienced engineers often understand projections by the method of body analysis. Main steps of the method are as follows: 1) A complex body is divided into parts by decomposing view-loops. 2) Corresponding loops selected from three views are validated their coherence according to rules of orthographic projection; 3) Corresponding 3D model are generated from typical patterns according to view loops and multiple interpretations are eliminated by views testing.

\subsection{Main idea and whole framework of the new algorithm}

This paper simulates the method of body analysis and presents a new algorithm of 3D reconstruction. Reconstruction process of a goal solid is similar to that of machine assembly. Figure 1 shows the whole framework of the new algorithm. The main steps are as follows: First, multiple views are separated and graphics feature information is extracted from dxf-type files. Second, engineering semantics and possible a priori knowledge are used to recognize components or primitives and the combined relationship between those. Third, sweeping representation is firstly used for components reconstruction. If unsuccessful, the approach algorithm is then arranged for 3D reconstruction. Provided that the two methods are not fit for this component, the component can be reconstructed by program module $\mathrm{A}$ based on primitives decomposition. Fourth, each of decomposed primitives is supposed to be among of elementary feature, sweeping feature, volumecutting feature and boundary feature. And it is reconstructed by using a corresponding algorithm in order of precedence, i.e. CSG $\rightarrow$ sweeping 
representation $\rightarrow$ the approach algorithm $\rightarrow$ B-Rep. Every reconstructed 3D component or primitive is validated by its projective views. Finally, all reconstructed components and primitives are assembled into a goal solid by Boolean operation and 3D editing operations.

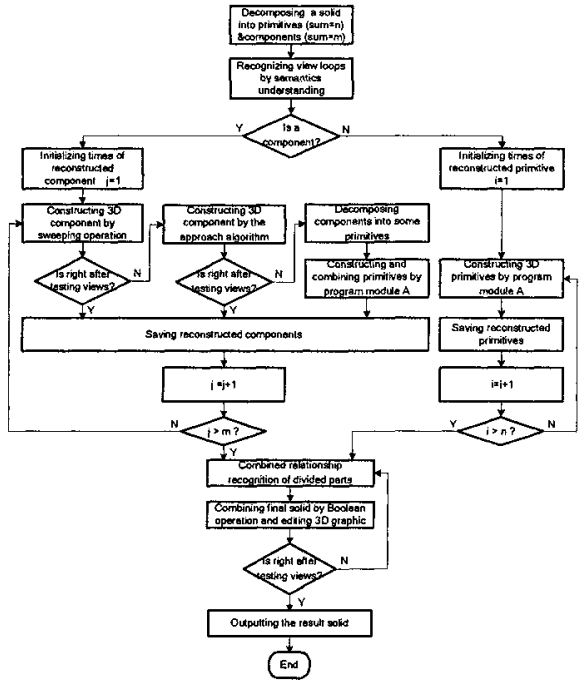

Figure I. Whole framework of the new algorithm



Figure 2. Framework graph of part reconstruction

\subsection{Partition and reconstruction of components /primitives}

In order to partition components and primitives, we take full advantage of the semantics of engineering drawings to accelerate primitive recognition. According to the process of the projection, the semantics are divided into expression mode semantics, projection relation semantics and dimension constraint semantics. Furthermore, every level is classified to explicit semantics and hidden semantics. Expression semantics understanding is the precondition of engineering drawings understanding; projection semantics understanding is the key process; understanding of dimension constraint semantics plays an assistant role. For example, the modeling information of sweeping-feature primitive, such as sectional loops and the sweeping height or path, is recognized through analyzing the explicit semantics and digging the hidden semantics. When multiple interpretations exist, views testing and visual reasoning are activated to pick out the most acceptable interpretation. Sectional views could also be involved to eliminate the unsuitable interpretations of projection views. From Fig.2, we can see that the new 
algorithm sets up a mechanism of supposing and ascertaining to reconstruct parts of the goal solid. It uses integrated reconstruction techniques. Different kinds of reconstruction algorithms are arranged to reconstruct different feature primitives according to their reconstruction efficiency from high to low. CSG is first used to reconstruct 3D primitives due to its highest reconstruction efficiency. But limited pre-defined primitive patterns restrict its applicability. So, every new reconstructed primitive is saved as a new pattern to enlarge the pattern base of CSG for feature recognition.

\section{IMPLEMENTATION}

The combination of ObjectARX 2002 and VC++6.0 is used to implement the new algorithm at the platform of AutoCAD 2004. Fig.3 shows the reconstructed solid of a mechanical part and its reconstructed parts. From it, we can see that it is divided into two components and two primitives by engineering semantics understanding. Fig.4 illustrates the reconstruction process of components and primitives reconstructed by different algorithms.

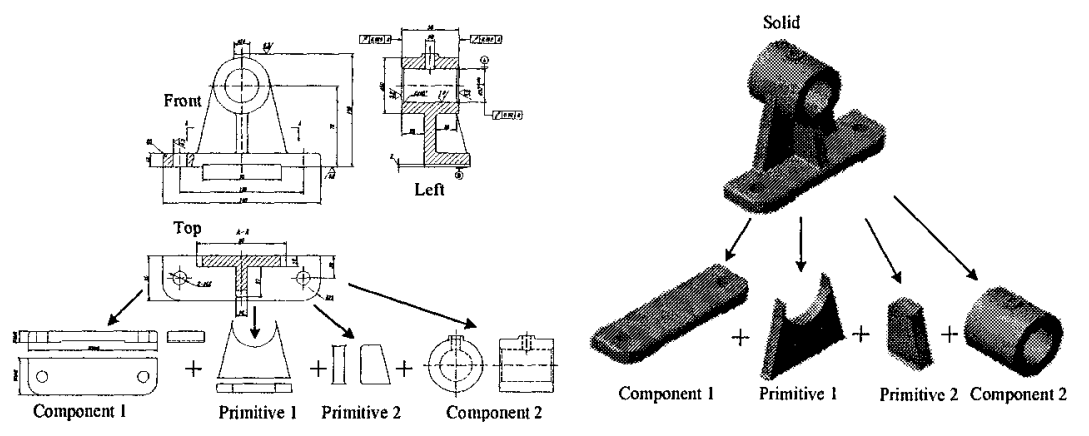

Figure 3. Original engineering drawings, its processed views of decomposed parts and reconstructed solid

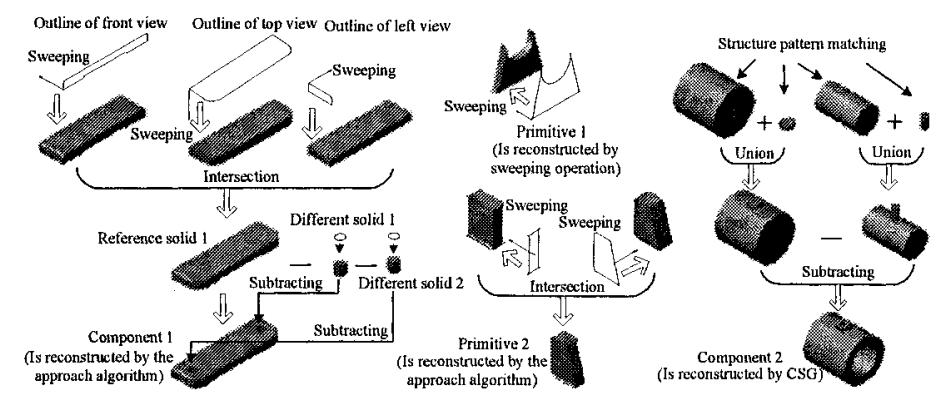

Figure 4. Diagram of reconstruction process of components and primitives 


\section{CONCLUSION}

In order to enhance the practicability of $3 \mathrm{D}$ reconstruction, this paper presents a new algorithm based on intelligent understanding engineering drawings. The characteristic of the algorithm is as follows: 1) complex body is divided into different kinds of components and primitives and engineering semantics is used to recognizing them and their combined relationships; 2) hypothesis and views testing are adopted to reconstruct and to validate the result solid; 3) integrated reconstruction techniques are used to enlarge the figuration domain of reconstructed objects; 4) case-based reasoning is used to establish a self-learning mechanism of feature recognition.

\section{REFERENCES}

1. Idesawa, M., (1973), A Systems to Generate a Solid Figure from Three View, Bulletin Of the JSME, Vol. 16(92), pp. 216-225.

2. Nagendra, I.V., and Gujar, V.G., (1988), 3D Objects from 2D Orthographic Views-A Survey, Computers \& Graphics, Vol. 12(1), pp. 111-114.

3. Preiss, K., (1984), Constructing the a Solid Representation from Engineering Projections, Computer and Graphics, Vol.8, pp. 381-389.

4. Aldefeld, B., (1983), On Automatic Recognition of 3-D Structures from 2D Representations, Computer-Aided Design, Vol. 15(2), pp. 59-64.

5. Chen, Z., and Perng, D.B., (1988), Automatic Reconstruction of 3D Solid Objects from 2D Orthographic Views, Pattern Recognition, Vol. 21(5), pp. 439-449.

6. Wang, W.D., and Grinstein, G.G., (1993), A Survey of 3D Solid Reconstruction from 2D Projection Line Drawings, Computer Graphics Forum, Vol. 12(2), pp.137-158.

7. Yoshiura, H., Fujimura, K. and Kunii, T.L., (1984), Top-down Construction of 3-D Mechanical Object Shape from Engineering Drawing, IEEE Compuder Magazine, Vol.(12), pp. 3240.

8. Joseph, S.H., and Pridmore, T.P., (1992), Knowledge-Directed Interpretation of Mechanical Engineering, IEEE Trans Pattern Analysis \& Machine Intelligence, Vol. 14(9), pp. 928-940.

9. Lu, G.D., and Peng, Q.S., (2000), Research on Primitive Relation Recognition Based on the Engineering Drawing Semantics, Journal of Computer Aided Design and Computer Graphics, Vol. 12(9), pp. 700-704 (in Chinese). 